\title{
Allergic rhinitis complicated by hypertrophy of the mucous membrane of nasal turbinates in patients of Northern Viet Nam
}

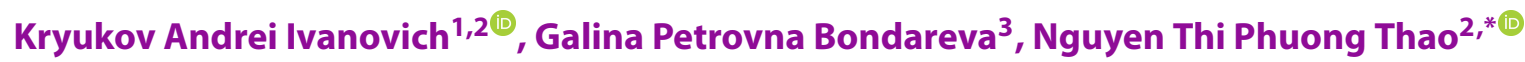

${ }^{1}$ L.I. Sverzhevskiy Research and Clinical Institute of Otorhinolaryngology, the Moscow Healthcare Department, Moscow, Russia

${ }^{2}$ Department of Otorhinolaryngology, Medical Faculty, N.I. Pirogov Russian National Research Medical University, Ministry of Health of the Russian Federation, Moscow, Russia

${ }^{3} \mathrm{NRC}$ Institute of Immunology FMBA of Russia, Moscow, Russia

\section{Correspondence}

Nguyen Thi Phuong Thao, Department of Otorhinolaryngology, Medical Faculty, N.I. Pirogov Russian National Research Medical University, Ministry of Health of the Russian Federation, Moscow, Russia

Email: phuongthao.ent@gmail.com

\section{History}

- Received: Jan 11, 2020

- Accepted: May 11, 2020

- Published: June 24, 2020

DOI : 10.15419/bmrat.v7i6.609

\section{Check for updates}

\section{Copyright}

(c) Biomedpress. This is an openaccess article distributed under the terms of the Creative Commons Attribution 4.0 International license.

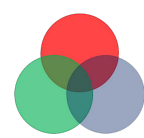

The Open Access Publisher

\begin{abstract}
Introduction: Constant contact with allergens contributes to the formation of allergic rhinitis and swelling of the mucous membrane of the nasal cavity. Moreover, constant inflammation stimulates hypertrophy of the mucous membrane of the nasal turbinates and paranasal sinuses. This study aimed to assess the clinical features of hypertrophic rhinitis (HR) in combination with allergic rhinitis (AR) in patients from Northern Vietnam to improve the effectiveness of treatment and quality of life of patients with this pathology of the nasal cavity. Methods: The study was conducted from June to Sptember 2018 by the Department of Otorhinolaryngology, Thai Nguyen Central General Hospital, Vietnam. A total of 158 patients with symptoms of chronic rhinitis, aged 5-70 years, were examined. All patients with allergic rhinitis were subjected to standard, specific allergological tests, such as the determination of specific IgE in the blood, and statistics were assessed according to the otorhinolaryngology examination and CT scan. All patients were also examined by otolaryngologists. Results: According to the results of our study, among 64 patients with a diagnosis of $\mathrm{AR}$, a total of $45(70.31 \%)$ patients were diagnosed as AR in combination with a hypertrophy of the mucous membrane of the nasal turbinate, with an average duration of morbidity of $5.8 \pm 1.6$ years. Moreover, 17 (37.78\%) patients were diagnosed with severe hypertrophy of the mucous membrane of the nasal inferior turbinate (the nasal inferior turbinate is $50 \%$ or more of nasal cavity and with nasal polyps). The sensitization to house dust mites and fungi was high. Conclusion: The peculiar findings of AR in combination with HR detected in patients of Northern Vietnam was associated with the onset of the disease at the age of 20-40 years, the predominance in men, the predominance of the disease in smokers, and the predominance in residents of cities and industrial areas (71.1\%). Indeed, there was an observed combination of AR with severe and moderate degree of HR. The sensitization of patients with AR in combination with HR in Northern Vietnam are similar to those in Asia, with predominant allergy to house dust mites.
\end{abstract}

Key words: allergic rhinitis, hyperactivity, plant pollen, hypertrophic rhinitis, hypertrophy, the determination of specific $\lg E$ in the blood

\section{INTRODUCTION}

Hypertrophic rhinitis (HR) is a chronic disease in which there is an increase (hypertrophy) of the mucous membrane and submucous layer of the nasal cavity, periosteum, and in the most advanced stages, bone tissue.

In severe cases, tissue changes are irreversible. In this regard, in most cases, patients require surgical treatment. Two places in the nasal cavity are most susceptible to aerodynamic loading: the front ends of the middle turbinate and the posterior ends of the inferior turbinate.

Depending on the degree of spread of the pathological process, HR can be divided into two forms- local and diffuse hypertrophy. With the local form of the disease, the lesions are concentrated in one place; however, in the absence of adequate treatment, the inflammatory process spreads and transforms into the diffused form ${ }^{1}$.

The cause of HR can be a chronic runny nose, prolonged hypothermia, contact with aeroallergens (household allergens, pollen, fungal allergens, etc.), infectious agents, tobacco, household and industrial dust, abuse of nasal vasoconstrictor drugs, endocrine system diseases, and/or nasal septum deformation ${ }^{2}$. A healthy mucous membrane copes well with external influences and there are no structural changes in it. In chronic inflammation of the nasal mucous membrane, as well as with an increase in aerodynamic load (curvature of the nasal septum and other violations of the anatomy of the nasal cavity), the compensatory growth of the mucous membrane is seen in the socalled "weak zones".

According to some studies, deformations of the nasal septum increase in the medial mucous membrane of the nasal turbinate by about $82 \%$, which subsequently worsens nasal breathing, causes breathing through the 
mouth, delayed secretion, snoring, and/or sleep apnea $^{3,4}$.

Epidemiological studies have found that the prevalence of all forms of allergic rhinitis (AR) is influenced by regional characteristics, such as those related to climate geography (e.g. temperature variability, air humidity, flora, and the social characteristics of the region $)^{5}$.

Vietnam is located in a tropical zone with a high average temperature; this region has a peculiar spectrum of aeroallergens and is characterized by the level of social development. For most patients, the cost of treating AR in combination with HR is significant. Thus, the diagnostic and therapeutic tactics employed by patients living in this territory can have some significant differences.

However, to date, there is very little research or information regarding the prevalence and clinical picture of this disease in Vietnam and, in particular, in its northern region.

Our study aims to study the clinical features of HR in combination with $\mathrm{AR}$ in patients of the Northern Vietnam region to improve the effectiveness of treatment and improve the quality of life of patients with AR with concomitant pathology of the nasal cavity.

\section{MATERIALS AND METHODS}

\section{Materials}

The study was conducted from June to September 2018 by the Department of Otorhinolaryngology, Thai Nguyen Central General Hospital, Vietnam. The field of study included urban and rural areas. An urban area was defined as having a population density (in 2018) from 400 to 3000 people per $\mathrm{km}^{2}$, and a rural area was defined as having a developing modern agricultural production and industrial plants. A total of 158 patients (aged 5 to 70 years) with chronic rhinitis (CR) were examined, of which 64 patients were identified and diagnosed as having AR.

\section{Methods}

All patients with AR were assessed for complaints of nasal congestion, olfactory disturbance, sneezing, itching (in the nasal cavity and eyes), rhinorrhea, and drainage of mucus along the back wall of the pharynx. In this study, the exclusion criteria were as follows: pregnancy or lactation, acute infectious pathologies, autoimmune diseases, oncopathology, psychosomatic disorders, acquired immunodeficiency syndrome (AIDS), treatment with antihistamines, use of glucocorticosteroids, and use of mast cell stabilizers.
All patients with AR underwent a standard specific and thorough allergological study, which included allergological history, the determination of specific IgE in the blood in response to house dust, pollen, and epidermal allergens (manufactured by NPO Microgen, Russia) and otorhinolaryngological examination. The study was approved by the Medical Ethics Research Committee of Thai Nguyen Central General Hospital, Vietnam. Informed consent was obtained from the patients before any tests or evaluations were conducted. The number of the ethical approval letter was BVTWTN2008201.

\section{Allergological history}

The interview questionnaire used was the recently developed questionnaire from the Russian Association of Allergologists and Clinical Immunologists ${ }^{6}$. Additional questions were taken from the Global Allergy and Asthma European Network (GA2LEN) questionnaire $^{7}$. The questionnaire of our study herein included questions mainly about respiratory and allergic symptoms, diagnoses, and smoking habits, such as the below:

- a. Allergic rhinitis (AR): "Do you have nasal allergy, including hay fever?"

- b. AR symptoms lasting 12 months: "Have you been troubled by nasal allergies in the last 12 months?"

- c. "When/what was the frequency of duration of nasal congestion (year, month or season)?"

- e. Definitions of possible risk factors including smoking habits have been described in detail previously. "Are you a smoker?"

- f. "What concomitant diseases (bronchial asthma, nasal polyp, etc.) do you have?"

- g. Patients carry out an anamnesis collection questionnaire with scores (Table 1). Patients are evaluated for nasal congestion, rhinorrhea, and olfactory disturbance. The severity of symptoms is noted as points. The presence of 7-9 points indicates a severe course of the disease.

\section{Otorhinolaryngological examination}

Otorhinolaryngological examination of ear- nosethroat (ENT) organs included the use of endoscopic techniques; rigid KarlStorz Gmb H \& Co. KG endoscopes (2.7 and $4.0 \mathrm{~mm}$ in diameter, and viewing angle of 0 and $30^{\circ}$ ) were used.

All patients underwent computed tomography (CT) scan of the nose and paranasal sinuses in the frontal (coronary) projection with a section thickness of 5 


\begin{tabular}{ll}
\hline Table 1: Symptom scores & Score \\
\hline Nasal Congestion & 3 \\
\hline Persistent nasal congestion & 2 \\
Periodic nasal congestion & 1 \\
Minor difficulty in nasal breathing & 0 \\
Free nasal breathing & \\
The severity of rhinorrhea & 3 \\
Permanent discharge from the nasal cavity & 2 \\
Periodic discharge from the nasal cavity & 1 \\
Moderate discharge from the nasal cavity & 0 \\
No nasal discharge & \\
Olfactory disturbance & \\
Anosmia & 3 \\
Hyposmia & 2 \\
Intermittent Hyposmia & 1 \\
No dysfunction & \\
Total & 0
\end{tabular}

mm by a Somatom Scope (Siemens AG CT system; Siemens, USA).

Based on the results of the study, the degree of hypertrophy of the mucous membrane of the nasal inferior turbinate was graded into 1,2 , or 3 on the basis of size of the inferior turbinate ${ }^{8}$ :

No edema: normal inferior turbinate.

- Grade 1: Inferior turbinate is $25 \%$ of nasal cavity

- Grade 2: Inferior turbinate is $50 \%$ of nasal cavity

- Grade 3: Inferior turbinate is $75 \%$ or more of nasal cavity

\section{Specific IgE in the blood test}

Specific IgE in the blood was determined for allergens, including house dust allergens (house dust mites- $D$. Pteronyssinus, D. Farinae, and Blomia tropicalis), an allergen from cockroaches, hay dust, molds (Aspergillus fumigatus, Alternaria alternata/tenuis, Cladosporium herbarum, and Penicillium notatum), and food allergens (shrimp, crab, squid, mackerel, sardines, tuna, beef, chicken, egg yolk, and vegetables (celery, mushrooms, onions, or soy)). The measured values could automatically be used to calculate the concentration (in $\mathrm{IU} / \mathrm{ml}$ ) of allergen-specific IgE in each band. The RIDA qLine Allergy test system and the 1-Viet panel (from R-Biopharm AG, Germany)were used with WHO-standards. A positive reaction was recorded if specific IgE blood was 0.7 $\mathrm{IU} / \mathrm{ml}$.

\section{Statistical analysis}

All statistical analyses were performed by a professional statistician using Microsoft Excel version 12.0.4518.1014 and SPSS Statistics 20. Descriptive statistics methods were used to evaluate the results.

To find the correlative relationship between the traits, the Friedman and Wilcoxon rank correlation methods were used; a p-value $<0.05$ was considered statistically significant. Demographic and exposure data were used as independent variables; these included sex, age, area, and smoking habits. The results were expressed as odds ratios (OR) with 95\% confidence intervals (CI).

\section{RESULTS}

According to the results of our study, among the 64 patients with a diagnosis of AR, a total of 45 (70.31\%) of patients were diagnosed as AR in combination with hypertrophy of the mucous membrane of the nasal turbinate, with an average duration of morbidity of 5.8 \pm 1.6 years.

Among the patients with AR in combination with $\mathrm{HR}$, 21 (46.7\%) were men and 24 (53.3\%) were women. The age distribution of the subjects of AR was: 1 (22.2\%) (under the age of 20 years), 25 (55.6\%) (aged 
20 to 40 years), 14 (31.3\%) (aged 40 to 60 years), and 5 (11.1\%) (aged 60 to 70 years). The average age of the patients was $46.15 \pm 20.54$ years, while half of the patients had a working age from 21 to 40 years.

In the AR with HR subjects, 32 (71.1\%) remained in urban districts, and 13 (28.9\%) were in rural districts. Furthermore, out of 45 of these patients, 11 (17.19\%) lived near industrial areas while $34(75.6 \%)$ lived in environmentally clean areas. Patients living in urban areas were more associated with AR prevalence than those in rural areas $(\mathrm{OR}=1.62)$.

In 16 (35.6\%) patients, all of whom were men, there was a history of smoking for 7-17 years (with 5 cigarettes up to 1 pack per day); 5 of these patients were diagnosed for the first time with polyps of the middle turbinate. Patients who were smokers had a significant risk of AR with $\mathrm{HR}$ incidence $(\mathrm{OR}=1.14)$. According to the history of patients, vasoconstrictor drugs (Naphazolin, Otrivin, etc.) were often used for long-term, as seen in 4 (8.9\%) patients, all of whom were men.

Rhinorrhea was the most prevalent symptom among the participants: $45(100 \%)$ patients complained of nasal congestion, 45 (100\%) had private onset sneezing, and 14 (31.11\%) had olfactory disturbance (Table 2 ).

Moreover, according to the otorhinolaryngology examination and computer tomography (CT) scan, among the total of 45 patients with AR in combination with HR, 17 (37.78\%) patients were diagnosed with severe hypertrophy of the mucous membrane of the nasal inferior turbinate (the nasal inferior turbinate is $50 \%$ or more of nasal cavity and with nasal polyps), while $28(62.22 \%)$ of them had moderate hypertrophy of the mucous membrane of the nasal inferior turbinate (the nasal inferior turbinate is from $25 \%$ to $50 \%$ of nasal cavity).

There was a relationship is shown between clinical examination and medical history, and between bad lifestyle and degree of hypertrophy of the mucous membrane of the nasal inferior turbinate $(\mathrm{p}=0.009)$. According to the results of determining the level of specific IgE in the blood (high level of general and specific IgE), isolated sensitization to ticks ( $D$. Pteronyssinus, D. Farinae, B. Tropicalis) was found in $44(97.78 \%)$ patients. Mold allergy (Aspergillus fumigatus, Alternaria alternata/tenuis, Cladosporium herbarum, Penicillium notatum) was detected in 11 (24.44\%) of all patients examined, $4(8.89 \%)$ patients had sensitization to feather allergen, $1(2.22 \%)$ to cat hair, $1(1.56 \%)$ to cereal pollen, $1(2.22 \%)$ to cockroaches, and $1(2.22 \%)$ to crabs. For other nonbacterial allergens, negative results were seen. Moreover, 7 conchotomy, 9 polypotomy, 8 septoplasty, and
2 operations were performed to remove the bulla of the middle nasal turbinate and 5 submucous vasectomies with lateroconchopexy of the nasal inferior turbinate in patients with AR in combination with $\mathrm{HR}$ and anatomical defects. After the operation, an antibiotic, anti-inflammatory course with local therapy was used. The resulting treatment effect were evaluated after 1.3 and 6 months.

\section{DISCUSSION}

In the past, there have been very few studies of HR on allergy. Our research indicates that the prevalence of AR with HR among men is the same as for women. While men may have more bad lifestyle habits (smoking, alcohol consumption, etc.), housework is a risk factor for women.

An increased risk of allergic diseases among people exposed to tobacco smoke has been reported ${ }^{9}$. Our study results also confirm that smoking is not only a risk factor associated with AR, but also with HR. The risk factors for AR with HR indicated in our study are the location of the patient, i.e. where they reside and where they work over a long period. Moreover, it was found that AR in combination with moderate and severe forms of $\mathrm{HR}$ is more common in patients aged 20 to 40 years.

Among patients living in an ecologically clean area, we were not able to identify patients with severe forms of HR. It was found that the majority of patients with AR complicated by severe and moderate forms of HR are townspeople and residents of industrial areas (71.1\% of patients). The rapid growth of the urban population and growth of industrial production in recent years promote increased air pollution. Residents of cities often use private transport, including motorcycles. Air pollution is a factor in damage to the nasal mucosa ${ }^{9}$; in addition, poor living conditions (including high humidity, mold, house dust mites, cockroaches, and several types of animal dandruff (as part of house dust)) can contribute to hyperactivity and sensitization of the upper respiratory tract mucosa ${ }^{10}$. Information on the prevalence of severe forms of $\mathrm{HR}$ in patients with AR in rural areas is consistent with published data ${ }^{11-13}$.

Our study is the first to reveal the features of the clinical manifestations of AR complicated by HR in patients of the Northern Vietnam region, which is most often manifested by nasal congestion. A correlation was found between the severity of HR and worsening nasal breathing with olfactory disturbance. Our results are consistent with studies by Shiomori et al. in $2007^{14}$. 


\begin{tabular}{|c|c|c|c|}
\hline Nasal Congestion & Score & Number of patients & Grade point average \\
\hline Persistent nasal congestion & 3 & 10 & $1.71 \pm 1.23$ \\
\hline Periodic nasal congestion & 2 & 12 & \\
\hline Minor difficulty in nasal breathing & 1 & 23 & \\
\hline Free nasal breathing & 0 & 0 & \\
\hline The severity of rhinorrhea & & & $2.34 \pm 0.46$ \\
\hline Permanent discharge from the nasal cavity & 3 & 17 & \\
\hline Periodic discharge from the nasal cavity & 2 & 22 & \\
\hline Moderate discharge from the nasal cavity & 1 & 6 & \\
\hline No nasal discharge & 0 & 0 & \\
\hline Olfactory disturbance & & & $1.11 \pm 0.54$ \\
\hline Anosmia & 3 & 0 & \\
\hline Hyposmia & 2 & 5 & \\
\hline Intermittent Hyposmia & 1 & 9 & \\
\hline No dysfunction & 0 & 31 & \\
\hline Total & & 45 & \\
\hline
\end{tabular}

\section{CONCLUSION}

The peculiar observation in our study of AR in combination with HR detected in patients of Northern Vietnam was associated with the onset of the disease (at the age of 20-40 years), the predominance in men, smokers, and residents of cities and industrial areas (71.1\%), and the observation of the disease accompanied with severe and moderate degree of HR. In our study, sensitization of patients with AR in combination with HR revealed predominant hypersensitivity to household allergens, ticks, and indoor fungi.

\section{ABBREVIATIONS}

AIDS: Acquired immunodeficiency syndrome

AR: Allergic rhinitis

CR: Chronic rhinitis HR: Hypertrophic

CT: Computer tomography

HR: Hypertrophic rhinitis

\section{ACKNOWLEDGMENTS}

This work was supported by the Thai Nguyen Central General Hospital, Vietnam, no under grant.

\section{AUTHOR'S CONTRIBUTIONS}

Kryukov A.I, Bondareva G.P and NTPT collected the data. NTPT conducted analysis and interpretation of data. NTPT drafted the first version. All authors edited the first draft. All authors reviewed, commented and approved the final draft.

\section{FUNDING}

Not applicable.

\section{AVAILABILITY OF DATA AND MATERIALS}

Data and materials used and/or analysed during the current study are available from the corresponding author on reasonable request.

\section{ETHICS APPROVAL AND CONSENT TO PARTICIPATE}

This study was conducted in accordance with the amended Declaration of Helsinki. The institutional review board (the Medical Ethics Research Committee of Thai Nguyen Central General Hospital, Vietnam) approved the study, and all participants provided written informed consent. The number of the ethical approval letter was BVTWTN2008201.

\section{CONSENT FOR PUBLICATION}

Not applicable. 


\section{COMPETING INTERESTS}

The authors declare that they have no competing interests.

\section{REFERENCES}

1. Datta RK, Ramya B, Vinay SR. Comparative study between partial inferior turbinectomy and submucosal diathermy for treatment of inferior turbinate hypertrophy due to allergic rhinitis. International Journal of Otorhinolaryngology and Head and Neck Surgery. 2018;4(2):362-367. Available from: http://dx.doi.org/10.18203/issn.2454-5929.ijohns20180425.

2. Segboer CL, Fokkens WJ, Terreehorst I, Drunen CM. Endotyping of non-allergic, allergic and mixed rhinitis patients using a broad panel of biomarkers in nasal secretions. PLoS One. 2018;13(7):1-15. PMID: 30048449. Available from: https: //doi.org/10.1371/journal.pone.0200366.

3. Smitha CB, Kiran B, John S, Kumar C. Comparison of partial inferior turbinectomy and submucosal diathermy for hypertrophied inferior turbinate in allergic rhinitis patients. Journal of Evolution of Medical and Dental Sciences. 2015;4(54):94579467. Available from: https://doi.org/10.14260/jemds/2015/ 1369.

4. Almehizia AA, AlEssa RK, Alwusaidi KM, Alzamil KA. Allergic rhinitis: Disease characteristics and coping measures in Saudi Arabia. PLoS One. 2019;14(6):1-16. PMID: 31242201. Available from: https://doi.org/10.1371/journal.pone.0217182.

5. Stevens WW, Grammer LC. Occupational rhinitis: an update. Current allergy and asthma reports. 2015;15(1):487. PMID: 25430949. Available from: https://doi.org/10.1007/s11882014-0487-8.

6. Federal clinical guidelines for the diagnosis of allergic diseases Moscow. 2015;p. 1-28. Available from: http://nrcii.ru/docs/ Klinicheskie_rekomendacii_po_diagnostike_AZ.pdf.

7. Bousquet J, Burney PG, Zuberbier T, Cauwenberge PV, Akdis CA, Bindslev-Jensen $C$, et al. GA2LEN (Global Allergy and Asthma European Network) addresses the allergy and asthma 490 'epidemic'. Allergy. 2009;64(7):969-977. PMID: 19392994. Available from: https://doi.org/10.1111/j.13989995.2009.02059.x.

8. Deenadayal DS, kumar MN, Sudhakshin P, Hameed S. Radiofrequency Reduction of Inferior Turbinates in Allergic and Non Allergic Rhinitis. Indian Journal Otolaryngology Head And Neck Surgery. 2014;66(11):231-236. PMID: 24533389. Available from: https://doi.org/10.1007/s12070-011-0445-.

9. Hwang BF, Jaakkola JJ, Lee YL, Lin YC, Guo YL. Relation between air pollution and allergic rhinitis in Taiwanese schoolchildren. Respiratory Research. 2006;7:23. PMID: 16469096. Available from: https://doi.org/10.1186/1465-99217-23.

10. Mosges R, Klimek L. Today's allergic rhinitis patients are different: new factors that may play a role. Allergy;62(2007):969975. PMID: 17686101. Available from: https://doi.org/10.1111/ j.1398-9995.2007.01440.x.

11. Eriksson J, Ekerljung L, Lotvall J, Pullerits T, et al. Growing up on a farm leads to lifelong protection against allergic rhinitis. Allergy. 2010;65:1397-1403. PMID: 20497148. Available from: https://doi.org/10.1111/j.1398-9995.2010.02397.x.

12. Filipiak B, Heinrich J, Schafer T, Ring J, Wichmann HE. Farming, rural lifestyle and atopy in adults from southern Germanyresults from the MONICA/KORA study Augsburg. Clinical \& Experimental Allergy. 2001;31:1829-1838. PMID 11737033. Available from: https://doi.org/10.1046/j.13652222.2001.01246.x.

13. Nicolaou N, Siddique N, Custovic A. Allergic disease in urban and rural populations: increasing prevalence with increasing urbanization. Allergy. 2005;60:1357-1360. PMID: 16197466. Available from: https://doi.org/10.1111/j.13989995.2005.00961.x.

14. Shiomori T, Udaka T, Hashida K, Fujimura T, Hiraki N, Ueda N, et al. Evaluation of quality of life in patient with allergic rhinitis. Journal of UOEH. 2007;29(2):159-167. PMID: 17582988. Available from: https://doi.org/10.7888/juoeh.29.159. 